\title{
CD163 expression in leukemia cutis
}

Background: Proper diagnosis of myeloid leukemia cutis (LG) is of great clinical importance but can be difficult because no single immunohistochemical marker is adequately sensitive or specific for definitive diagnosis. Thus, a broader panel of markers is often desirable. CD163 is highly specific for normal and neoplastic cells of the monocyte/histiocyte lineage. In this study, we examined the value of CD163 in the diagnosis of acute myeloid LC.

Methods: A total of 34 cases, including 18 cases of myelomonocytic or monocytic LC, 10 cases of myeloid LC without monocytic component and 6 cases of acute lymphoblastic leukemia/lymphoma (ALL), were stained with CD163.

Results: CD163 was expressed in 8 of 18 (44\%) of myelomonocytic or monocytic LC and 1 of $10(10 \%)$ of other myeloid LC, but in none of the ALL cases (0/6). CD163 was highly specific (90\%) for myeloid LC with a monocytic component, but showed low sensitivity in the diagnosis of both myeloid LC in general (24\%) and myeloid LG with a monocytic component (44\%).

Conclusions: Our results suggest that GD163 has utility as a specific marker for myeloid LC in conjunction with currently used immunohistochemical stains, but should not be used alone for diagnosis.

Harms PW, Bandarchi B, Ma L. CD163 expression in leukemia cutis. J Gutan Pathol 2010; 37: 953-957. (C) 2010 John Wiley \& Sons A/S.

\section{Paul W. Harms ${ }^{1}$, Bizhan Bandarchi' ${ }^{2}$ and Linglei $\mathrm{Ma}^{1,3}$}

${ }^{1}$ Department of Pathology, University of Michigan Medical Center, Ann Arbor, MI, USA,

${ }^{2}$ Department of Applied Molecular Oncology, University of Toronto, Princess Margaret Hospital and Ontario Cancer Institute, Toronto, Canada, and ${ }^{3}$ Department of Dermatology, University of Michigan Medical Center, Ann Arbor, MI, USA

Linglei Ma, MD, PhD,

Department of Pathology, Dermatopathology

Division, University of Michigan, M3261,

Medical Sciences 1, 1301 Catherine St., Ann

Arbor, Ml 48109-0602, USA

Tel: +17347644460

Fax: +17347644690

e-mail: lingleim@umich.edu

Accepted for publication January 9, 2010
Acute myeloid leukemia (AML) may form tumor masses in extramedullary sites, with skin being one of the most common sites. ${ }^{1}$ The involvement of skin by myeloid leukemic infiltrate, or myeloid leukemia cutis (LC), may precede the appearance of systemic disease, occur concurrently with systemic AML, or herald disease recurrence in treated patients. ${ }^{1,2}$. In addition, the onset of myeloid LC may be a sign that blastic transformation of a pre-existing myelodysplastic syndrome or myeloproliferative neoplasm (previously known as chronic myeloproliferative disease) is occurring.

Recognition of myeloid LG is clinically essential, yet sometimes poses diagnostic difficulties. Particularly, the diagnosis of LC in the absence of known systemic disease (aleukemic LC), which occurs in $7 \%$ of cases is challenging. ${ }^{2}$ Clinical manifestations of myeloid LC are non-specific and may present as erythematous rash, urticaria, purpura, plaques or nodules. Histopathologically, early myeloid LC may involve the dermis in an angiocentric pattern, with or without infiltration between collagen bundles; more developed lesions show diffuse dermal infiltrate of immature granulocytes or monocytes, sparing the epidermis. $^{2}$ In many cases, however, histopathologic features alone are not sufficient to rule out other entities, such as cutaneous lymphoproliferative processes or acute lymphoblastic leukemia/lymphoma (ALL). ${ }^{3}$ Therefore, immunophenotyping is routinely employed for accurate diagnosis of LC. Useful markers include CD68 (KP1 or PG-M1), myeloperoxidase (MPO), CD117, CD43 and lysozyme, as well as B-cell or T-cell markers. CD68, one of the most sensitive markers, may also stain a variety of other tumor types, including melanomas and carcinomas., ${ }^{4,5}$ MPO is more specific for myeloid lineage, but reports vary regarding its sensitivity, particularly in LC with monocytic differentiation. ${ }^{1-3}$ Rarely, myeloid LC may display aberrant expression of $\mathrm{B}$-cell or T-cell markers. ${ }^{3}$ In challenging cases, a more complete armamentarium of immunohistochemical markers is desirable in order to increase diagnostic accuracy. 
CD163 is a transmembrane glycoprotein in the class B scavenger receptor cysteine-rich (SRCR) family. CD163 exhibits high-affinity binding and endocytosis of the haptoglobin-hemoglobin complex, thus serving as the primary effector of haptoglobin-hemoglobin clearance from circulation. ${ }^{6}$ CD163 expression is largely restricted to the monocyte/macrophage lineage. ${ }^{4,5}$ In bone marrow and lymph nodes, CD163 immunoreactivity is detected in AMLs with monocytic differentiation. Carcinomas, most melanomas and B-cell or T-cell lymphomas were found to be negative for CD163, ${ }^{4,5,7,8}$ although its expression has been reported in $29 \%$ of desmoplastic melanomas. ${ }^{9}$

Few reports have examined the expression of CD163 in myeloid LC, and none evaluated its specificity in distinguishing myeloid LG from cutaneous
ALL. ${ }^{4,10,11}$ Because of its specificity for the monocytic lineage and its expression in bone marrow and extramedullary sites other than skin, we explored the expression of CD163 in myeloid LG. We hypothesized that GD163 would be useful as a specific marker for myeloid LG with monocytic differentiation.

\section{Materials and methods}

After obtaining approval from the University of Michigan Institutional Review Board for human subject research, 34 consecutive cases of LC were identified through a retrospective search of the pathology database from the Department of Pathology at the University of Michigan. All tumors examined are listed in Table 1.

Table 1. CD163 staining results for LC in comparison to other markers

\begin{tabular}{|c|c|c|c|c|c|c|}
\hline Case & Diagnosis & CD163 & CD68 & Lysozyme & MPO & CD43 \\
\hline 1 & AML-MO & Negative & ND & ND & ND & Positive \\
\hline 2 & AML-M1 & Negative & ND & ND & Rare positive & ND \\
\hline 3 & AML-M1 & Negative & ND & Negative & Focal positive & Positive \\
\hline 4 & AML-M1 & Negative & ND & Negative & Rare positive & Positive \\
\hline 5 & AML-M1 & Negative & Negative & Negative & Negative & Weak positive \\
\hline 6 & AML-M2 & $>50 \%$ & ND & ND & ND & Positive \\
\hline 7 & AML-M2 & Negative & ND & Positive & Positive & Positive \\
\hline 8 & AML-M2* & Negative & ND & ND & ND & ND \\
\hline 9 & AML-M3v' ${ }^{\dagger}$ & Negative & ND & ND & ND & ND \\
\hline 10 & AML-M4 & $>50 \%$ & Positive & Subset positive & Subset positive & Positive \\
\hline 11 & AML-M4 & $>50 \%$ & Positive & Positive & Subset positive & Positive \\
\hline 12 & AML-M4 & $10-50 \%$ & ND & ND & Subset positive & Positive \\
\hline 13 & AML-M4 & $10-50 \%$ & ND & Subset positive & Positive & Positive \\
\hline 14 & AML-M4 & Negative & ND & ND & Rare positive & Positive \\
\hline 15 & AML-M4 & Negative & Positive & Negative & Negative & Positive \\
\hline 16 & AML-M4 & Negative & Positive & Positive & Rare positive & Positive \\
\hline 17 & AML-M4 & Negative & Positive & Positive & Subset positive & Positive \\
\hline 18 & AML-M4 & Negative & ND & Subset positive & Subset positive & Positive \\
\hline 19 & AML-M4 & Negative & Weak positive & ND & Negative & Positive \\
\hline 20 & AML-M4 & Negative & Positive & Subset positive & ND & ND \\
\hline 21 & AML-M5b & $>50 \%$ & ND & Positive & Subset positive & Positive \\
\hline 22 & AML-M5 & $10-50 \%$ & ND & Negative & Negative & Positive \\
\hline 23 & AML-M5 & $10-50 \%$ & Weak positive & Positive & Rare positive & Positive \\
\hline 24 & AML-M5 & Negative & Negative & Subset positive & Positive & Positive \\
\hline 25 & AML-M5b & Negative & ND & ND & ND & ND \\
\hline 26 & AML-M5b & Negative & Weak positive & Positive & Rare positive & Positive \\
\hline 27 & AML-M4/M5 & $10-50 \%$ & Negative & Positive & Negative & Positive \\
\hline 28 & AML-M6 & Negative & ND & ND & ND & ND \\
\hline 29 & T-ALL & Negative & ND & ND & ND & ND \\
\hline 30 & Precursor B-ALL & Negative & ND & Subset positive & Negative & Positive \\
\hline 31 & Precursor B-ALL & Negative & ND & ND & ND & ND \\
\hline 32 & Precursor T-ALL & Negative & Negative & Negative & Negative & Weak positive \\
\hline 33 & T-ALL & Negative & ND & ND & ND & ND \\
\hline 34 & Precursor B-ALL & Negative & ND & ND & ND & ND \\
\hline
\end{tabular}

MP0, myeloperoxidase; AML, acute myeloid leukemia; ALL, acute lymphoblastic leukemia/lymphoma; ND, not done.

*AML, $\mathrm{t}(8 ; 21)(\mathrm{q} 22 ; \mathrm{q} 22)$ under the new WHO classification.

${ }^{\dagger}$ Acute promyelocytic leukemia under the new WHO classification. 


\section{CD163 expression in leukemia cutis}

Immunohistochemical stains other than CD163 were performed prior to inclusion of cases in the study. The original diagnoses were confirmed by histopathologic review by two pathologists $(\mathrm{PH}$ and LM). All AML cases collected for this study were from our pathology archives and were classified using the French-American-British (FAB) system at the time of the original diagnoses. Therefore, we have utilized this classification in the current study. According to the current World Health Organization (WHO) guidelines, ${ }^{1}$ we found that the majority of cases would fall under the category of AML, not otherwise specified. There were two exceptions: one case of AML-M3v fits best as acute promyelocytic leukemia and one AML-M2 would be classified as AML with $\mathrm{t}(8 ; 21)(\mathrm{q} 22 ; \mathrm{q} 22)$ under the new WHO guidelines (see Table 1).

The unstained slides were stained with CD163 antibody, as previously described. ${ }^{9}$ Briefly, $4 \mu \mathrm{m}$ thick unstained sections were pretreated with Ventana CG1 solution ( $\mathrm{pH}$ 8.0) for antigen retrieval and were incubated with NCL-CD163 monoclonal antibody (clone 10D6, 1:50 dilution; Vision Biosystems, Norwell, MA, USA) using a Ventana Benchmark XT system (Ventana Medical Systems, Tucson, AZ, USA). The UltraVision System and a 3,3'-diaminobenzidine chromagen were used for detection.

The staining pattern and intensity of CD163 were assessed on each slide. Cytoplasmic and/or membranous staining for CD163 in at least 10\% of tumor cells was considered positive. Entrapped dendritic cells or macrophages were not considered when determining positivity. The percentage of tumor cells labeled was recorded as one of three categories: $<10 \%$ (negative), $10-50 \%$ and $>50 \%$.

\section{Results}

As previously described, CD163 labeled dermal dendritic cells in normal skin (Fig. 1A). ${ }^{9,12}$ The results of CD163 staining in LC cases are summarized in Tables 1 and 2. In many cases, additional markers for
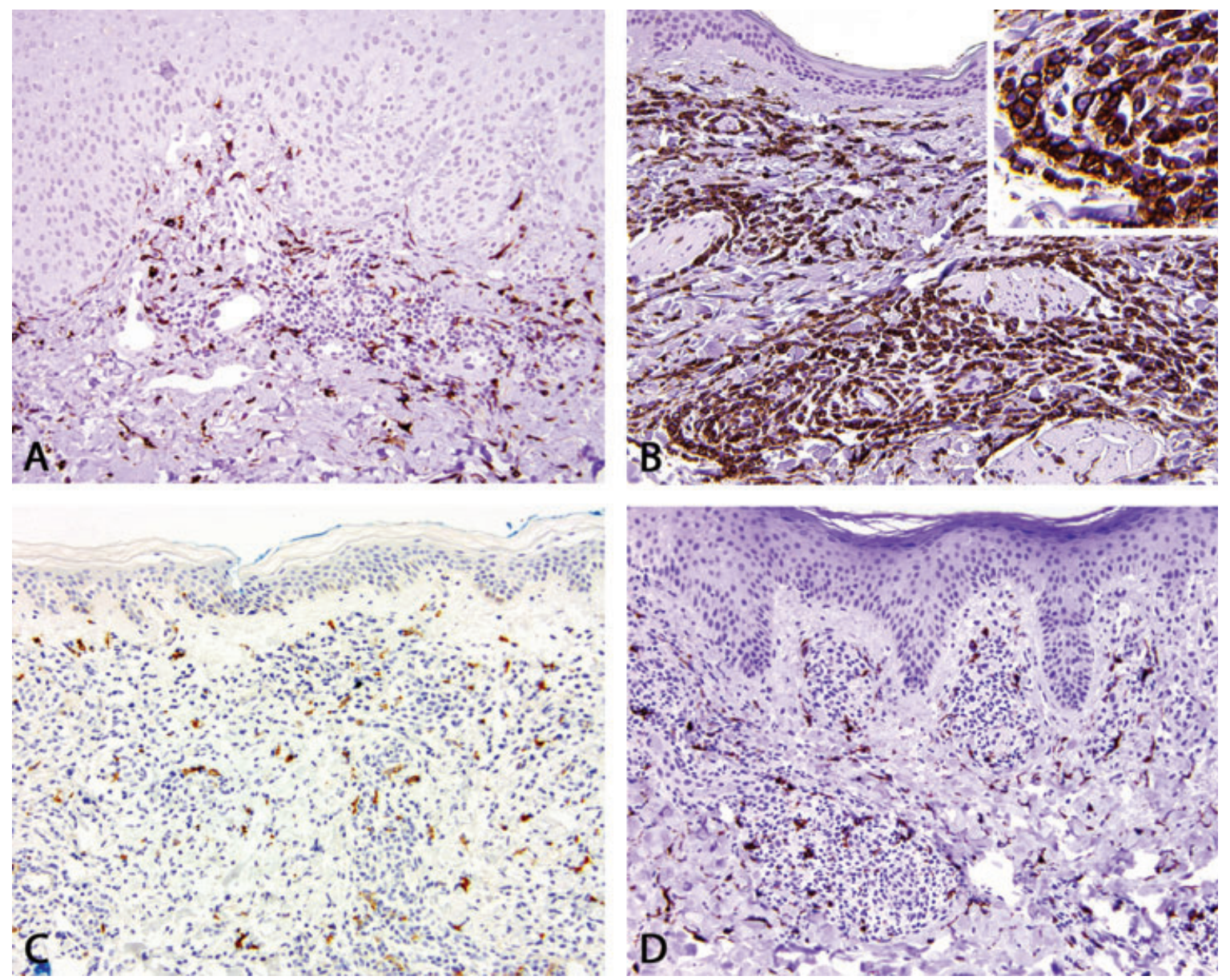

Fig. 1. CD163 expression in leukemia cutis (LC). A) CD163 labels dermal dendritic cells in normal skin. B) CD163 expression in myeloid LG with monocytic differentiation. G) Myeloid LG without monocytic differentiation normally does not express CD163. D) CD163 is absent in LC associated with ALL. 
Table 2. Summary of immunohistochemistry results in LC by various markers*

\begin{tabular}{lccccc}
\hline Diagnosis & CD163 & CD68 & Lysozyme & MP0 & CD43 \\
\hline AML-M4 & $4 / 11(36 \%)$ & $7 / 7$ & $7 / 8$ & $8 / 10$ & $10 / 10$ \\
AML-M5 & $3 / 6(50 \%)$ & $2 / 3$ & $4 / 5$ & $4 / 5$ & $5 / 5$ \\
AML-M4-5 & $8 / 18(44 \%)$ & $9 / 11$ & $12 / 14$ & $11 / 16$ & $16 / 16$ \\
AML, other & $1 / 10(10 \%)$ & $0 / 1$ & $1 / 4$ & $4 / 5$ & $6 / 6$ \\
$\quad$ than M4-5 & & & & & \\
AML, all & $9 / 28(32 \%)$ & $9 / 12$ & $13 / 18$ & $15 / 21$ & $22 / 22$ \\
ALL & $0 / 6(0 \%)$ & $0 / 1$ & $1 / 2$ & $0 / 2$ & $2 / 2$ \\
\hline
\end{tabular}

MPO, myeloperoxidase; AML, acute myeloid leukemia; ALL, acute lymphoblastic leukemia/lymphoma.

*For all listed markers, numbers of positive cases are given as a fraction over total cases stained.

myeloid LC were used alongside CD163 (Tables 1 and 2).

Among cases of myeloid LC, CD163 positivity was observed in $44 \%(8 / 18)$ of myeloid LCs with a monocytic component (AML-M4 or AML-M5 in the FAB classification system) (Fig. 1B). AML-M5 showed a higher probability of CD163 expression compared with AML-M4 (50 and 36\% positivity, respectively). Among positive cases, there was no significant difference between AML-M4 and AMLM5 with respect to staining pattern or intensity. With one exception (1/10), CD163 was negative in myeloid LC without a monocytic component (FAB classification of AML M0-M3 or M6) (Fig. 1C). Of note, one case of AML-M2 showed strong and diffuse CD163 positivity. In addition, all six cases of $\mathrm{T}$ ALL or B-ALL were negative for CD163 expression (Fig. 1D).

In our dataset, CD163 was 100\% specific for the purpose of distinguishing myeloid LC from LC associated with ALL. The expression of CD163 was also highly specific $(90 \%)$ for myeloid LG with a monocytic component when compared with myeloid LC without monocytic differentiation. CD163 showed low sensitivity (24\%) for all myeloid LC and a sensitivity of $44 \%$ for myeloid LG with a monocytic component.

\section{Discussion}

Myeloid LC often poses a diagnostic challenge, particularly in cases of aleukemic LC. Because morphologic features are rarely adequate for diagnosis, a precise immunophenotyping is critical. Standard markers for this purpose include CD68, MPO, lysozyme or CD43. As the most common types of myeloid LC are AML-M4 or AML-M5, finding a reliable monocytic marker will be of particular utility. The reported sensitivity of CD68 (KP1 or PG-M1) for AML varies in the literature from 33 to $100 \% .4,11,13,14$ Although PGM1 is regarded as more specific than KP1, both have been shown to stain melanomas and other malignancies. ${ }^{4}$ In addition, they often stain AMLs without a monocytic component. ${ }^{4,11,13}$ Although highly specific for the myeloid lineage, MPO has been reported to be only $58 \%$ sensitive for all myeloid LC, ${ }^{2}$ and is negative in many cases with monocytic differentiation. ${ }^{1,3,4}$ This is to be expected as MPO is generally strongly expressed only in leukemias with a prominent granulocytic component (i.e. AML-M2, AML-M3 and AMLM4). The monocyte/granulocyte marker lysozyme is highly sensitive, with expression in $93 \%$ of myeloid sarcomas; ${ }^{15}$ however, it is frequently negative in immature monoblasts. ${ }^{1-3,7,13,14,16,17}$ CD43 is sensitive for AML, but is also expressed in a large percentage of B-cell and T-cell lymphomas. ${ }^{1,18}$ Ultimately, because no marker or combination of markers is completely sensitive or specific, additional markers are often desirable to increase diagnostic confidence in cases of myeloid LC.

CD163 is an endocytic receptor responsible for scavenging haptoglobin-hemoglobin complexes ${ }^{6}$ and its expression is largely restricted to cells of the monocytic/histiocytic lineage. ${ }^{5}$ Recent studies showed the expression of CD163 in tumors derived from monocytes/histiocytes, including AML with monocytic differentiation. ${ }^{4,5}$ Moreover, Garcia et al. showed that CD163 was less sensitive (49\%) than CD68 (81\%), but was slightly more specific (89\%) than CD68 (83\%) for the presence of monocytic differentiation in AMLs of bone marrow. ${ }^{7}$ Recently, Cronin et al. presented a large study of CD163 in myeloid $\mathrm{LC}^{11}$ and reported $25 \%$ sensitivity for myeloid LC. Among seven positive cases, three were acute monocytic leukemia and two were associated with chronic myelomonocytic leukemia. In agreement with their observations, we found that the overall sensitivity of CD163 for myeloid LC in general was $32 \%$. The slight difference in sensitivity between Cronin et al.'s study and ours could be because of the higher proportion of cases with monocytic differentiation in our study group.

We showed that CD163 had a sensitivity of $44 \%$ for detecting myeloid LC with monocytic differentiation, which correlates well with Garcia et al.'s findings. ${ }^{7}$ The sensitivity we observed is lower than the sensitivity $(60 \%)$ reported by Cronin et al., in part because those authors included only acute monocytic leukemia in the calculation. ${ }^{11}$ Moreover, we found that CD163 was slightly more likely to be expressed in AML-M5 than AML-M4, probably as a result of greater monocytic component in the AML-M5. Similarly, a previous study in leukemic blasts from peripheral blood has shown that CD163 is more frequently expressed in leukemic cells 


\section{CD163 expression in leukemia cutis}

of AML-M5 than AML-M4, although this study showed a much greater difference between the two (84 and 25\% , respectively) than ours. ${ }^{8}$

Interestingly, we found 1 of 10 myeloid LCs without monocytic differentiation stained strongly for CD163. CD163 immunoreactivity has been previously reported in occasional AMLs without monocytic differentiation. ${ }^{7,8,11}$ Flow cytometry of bone marrow in this case showed a CD13+/CD14+ monocytic cell population. As monocytic differentiation is associated with a greater propensity for extramedullary tumor formation in AML, it is possible that the skin infiltrate in this case was enriched for cells with monocytic differentiation.

As indicated in Table 2, no single marker was positive in all cases of myeloid LC. Given the high specificity of CD163 for myeloid LG with monocytic differentiation, as well as the observation that no marker is $100 \%$ sensitive, an optimal approach toward immunophenotyping potential cases of myeloid LC would be to combine CD163 with CD68, MPO, lysozyme and CD43 (with additional markers as needed), in order to maximize both specificity and sensitivity.

It is important to note that CD163 may be expressed in other diseases, including Langerhans cell histiocytosis. ${ }^{9}$ A true diagnostic dilemma arises when attempting to distinguish myeloid LC from histiocytoid Sweet's syndrome. ${ }^{19}$ Our preliminary data (unpublished observation) indicates that GD163 may not be helpful for this purpose. The finding is unsurprising, as CD163 does not distinguish between mature and immature cells of the monocytic/histiocytic lineage.

In summary, CD163 represents an important addition to an immunohistochemical panel for diagnosing myeloid LC with monocytic differentiation, given its high specificity for cells of monocytic lineage. However, CD163 is only modestly sensitive for myeloid LC and therefore should not be used alone for diagnosis.

\section{References}

1. Arber DA, Brunning RD, LeBeau MM, et al. Acute myeloid leukaemia and related precursor neoplasms. In Swerdlow SH, Campo E, Harris NL, Jaffe ES, Pileri SA, Stein H, Thiele J, Vardiman JW, eds. WHO classification of tumours of haematopoietic and lymphoid tissues. Lyon, France: International Agency for Research on Cancer, 2008; 109.

2. Cibull TL, Thomas AB, O’Malley DP, Billings SD. Myeloid leukemia cutis: a histologic and immunohistochemical review.J Cutan Pathol 2008; 35(2): 180

3. Audouin J, ComperatE, Le Tourneau A, et al. Myeloid sarcoma: clinical and morphologic criteria useful for diagnosis. Int J Surg Pathol 2003; $11(4): 271$.
4. Lau SK, Chu PG, Weiss LM. CD163: a specific marker of macrophages in paraffin-embedded tissue samples. Am J Clin Pathol 2004; 122(5): 794.

5. Nguyen TT, Schwartz EJ, West RB, Warnke RA, Arber DA, Natkunam Y. Expression of CD163 (hemoglobin scavenger receptor) in normal tissues, lymphomas, carcinomas, and sarcomas is largely restricted to the monocyte/macrophage lineage. Am J Surg Pathol 2005; 29(5): 617.

6. Kristiansen M, Graversen JH, Jacobsen C, et al. Identification of the haemoglobin scavenger receptor. Nature 2001; 409(6817): 198.

7. Garcia C, Gardner D, Reichard KK. CD163: a specific immunohistochemical marker for acute myeloid leukemia with monocytic differentiation. Appl Immunohistochem Mol Morphol 2008; 16(5): 417.

8. Walter RB, Bachli EB, Schaer DJ, Ruegg R, Schoedon G. Expression of the hemoglobin scavenger receptor (CD163/ HbSR) as immunophenotypic marker of monocytic lineage in acute myeloid leukemia. Blood 2003; 101(9): 3755.

9. Pouryazdanparast P, Yu L, Cutlan JE, Olsen SH, Fullen DR, Ma L. Diagnostic value of CD163 in cutaneous spindle cell lesions. J Cutan Pathol 2008; 36: 859.

10. Alexiev BA, Wang W, Ning Y, et al. Myeloid sarcomas: a histologic, immunohistochemical, and cytogenetic study. Diagn Pathol 2007; 2: 42.

11. Cronin DM, George TI, Sundram UN. An updated approach to the diagnosis of myeloid leukemia cutis. Am J Clin Pathol 2009; 132(1): 101.

12. Zaba LC, Fuentes-Duculan J, Steinman RM, Krueger JG, Lowes MA. Normal human dermis contains distinct populations of CD11c + BDCA-1+ dendritic cells and CD163 + FXIIIA+ macrophages. J Clin Invest 2007; $117(9): 2517$.

13. Kaddu S, Zenahlik P, Beham-Schmid C, Kerl H, Cerroni L. Specific cutaneous infiltrates in patients with myelogenous leukemia: a clinicopathologic study of 26 patients with assessment of diagnostic criteria. J Am Acad Dermatol 1999; 40(6 Pt 1): 966.

14. Sepp N, Radaszkiewicz T, Meijer CJ, et al. Specific skin manifestations in acute leukemia with monocytic differentiation. A morphologic and immunohistochemical study of 11 cases. Cancer 1993; 71(1): 124.

15. Chen J, Yanuck RR, 3rd, Abbondanzo SL, Chu WS, Aguilera NS. c-Kit (CD117) reactivity in extramedullary myeloid tumor/granulocytic sarcoma. Arch Pathol Lab Med 2001; 125(11): 1448.

16. Roth MJ, Medeiros LJ, Elenitoba-Johnson K, Kuchnio M, Jaffe ES, Stetler-Stevenson M. Extramedullary myeloid cell tumors. An immunohistochemical study of 29 cases using routinely fixed and processed paraffin-embedded tissue sections. Arch Pathol Lab Med 1995; 119(9): 790.

17. Traweek ST, Arber DA, Rappaport H, Brynes RK. Extramedullary myeloid cell tumors. An immunohistochemical and morphologic study of 28 cases. Am J Surg Pathol 1993; 17(10): 1011.

18. Lai R, Weiss LM, Chang KL, Arber DA. Frequency of CD43 expression in non-Hodgkin lymphoma. A survey of 742 cases and further characterization of rare CD43+ follicular lymphomas. Am J Clin Pathol 1999; $111(4)$ : 488.

19. Requena L, Kutzner H, Palmedo G, et al. Histiocytoid Sweet syndrome: a dermal infiltration of immature neutrophilic granulocytes. Arch Dermatol 2005; 141(7): 834. 